\title{
Comparing the Anxiety and Depression of Death between Chemical and Non-Chemical Veterans of Sardasht City, Iran
}

\section{ART ICLE INF O}

\section{Article Type}

Descriptive Study

\section{Authors}

Soleymani Sh. ${ }^{1} M A$,

Shaker Dioulagh A.* $P h D$

How to cite this article
Soleymani Sh, Shaker Dioulagh
A.Comparing the Anxiety and De-
pression of Death between Che-
mical and Non-Chemical Vetera-
ns of Sardasht City, Iran. Iranian
Journal of War \& Public Health.
2017;9(2):73-78.

* Psychology Department, Humanities Faculty, Urmia Branch, Islamic Azd University, Urmia, Iran

${ }^{1}$ Psychology Department, Humanities Faculty, Urmia Branch, Islamic Azd University, Urmia, Iran

\section{Correspondence}

Address: Psychology Department, 3rd Floor, Faculty of Humanities, Urmia Branch, Islamic Azad University, Basij Boulvared, Urmia, Iran Phone: +98 (44) 33688119 Fax: +98 (44) 33688119 ali.shaker2000@gmail.com

\section{Article History}

Received: November 12, 2016

Accepted: January 18, 2017

ePublished: April 24, 2017

\section{A B S T R A C T}

Aims Exposed to the war and chemical weapons, the veterans experience severe mental reactions. One of such experiences is the death coming feeling that finally leads to some disorders such as death depression and death anxiety. The aim of the study was to compare the death depression and anxiety in the chemically-injured and non-chemically-injured veterans in Sardasht Township.

Instrument \& Methods In the comparative-causal descriptive study, 100 veterans including 50 chemically-injured and 50 non-chemically-injured veterans of Sardasht Township were studied in 2016. The subjects were selected via available sampling method. Data was collected by the death anxiety index and the death depression questionnaire. Data was analyzed by SPSS 16 software using MANOVA and independent T test.

Findings The mean score of death anxiety in the chemically-injured veterans was significantly higher than the non-chemically-injured veterans $(p=0.001)$. The mean scores of death hoplessness, death failure, and total depression score in the chemically-injured veterans were significantly higher then the non-chemically-injured veterans $(p=0.001)$. Nevertheless, the mean scores of loneliness and death acceptance were not significantly different in the groups, respectively ( $\mathrm{p}>0.05)$.

Conclusion The levels of death anxiety and death depression in the chemically-injured veterans of Sardasht Township were higher than the non-chemically-injured veterans.

Keywords Death; Anxiety; Depression; Veterans

\section{I T A T I O N L I N KS}

[1] An investigation the relationship between post traumatic growth and attachment styles, stress coping styles \& quality of life in veterans with post-traumatic ... [2] Post-traumatic stress disorder in male chemical injured war veterans compared to non-chemical ... [3] Association of psychological symptoms and self esteem in chemical warfare agent exposed ... [4] Psychological health status of mustard gas exposed ... [5] The effect of war related PTSD on wife's marital satisfaction, mental health and children's behavioral ... [6] Death [7] Towards a definition of death [8] Psychiatric Disoders in ... [9] Spiritual effect of group therapy on depression, anxiety, death and belief in life after death continue to rely on the reports of the death of the [10] Existential... [11] Surveying the relationship between hope, death, anxiety with mental health on students of urmia ... [12] The relation between death anxiety and level of self-esteem ... [13] The Prediction of Death Obsession According to Religiosity Orientations ... [14] Psychiatric symptoms in chemical and somatic combat ... [15] Mental health in group of war veterans and their spouses in ... [16] Study of sleep quality in chemical-warfare-agents exposed ... [17] Study of the prevalence and intensity of depression in 100 devotees with chemical and non-chemical war injuies (30-70\%) of Imposed war ... [18] Relationship of mustard agent and lewisite exposure to psychological dysfunction. In: Veterans at risk: The health effects of mustard gas and skills training program on swimming performance and positive psychological ... [19] Effectiveness of short-term specialize inpatienttreatment for war-related posttraumatic stress disorder: A role for advanture-based counseling and .. [20] Anxiety, depression, and posttraumatic stress in iranian ... [21] A survey of death anxiety among personnel of a hospital ... [22] Differing death scenarios: self- esteem and Death ... [23] Psychometric properties of the Spanish form of Templer's Death Anxiety ... [24] Death Depression scale validation in ... [25] The effectiveness of the treatment plan wives of veterans with PTSD impact on reducing symptoms of this disorder in ... 


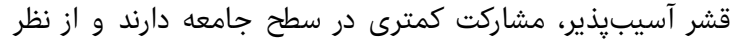

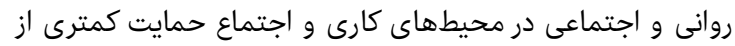

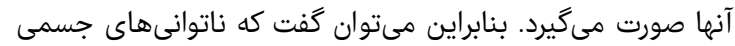

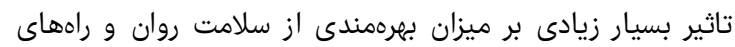

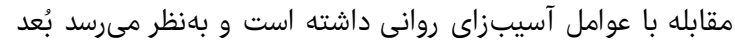

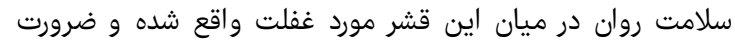

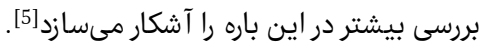

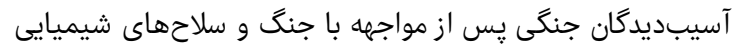

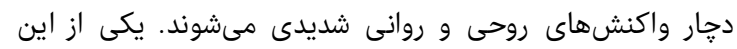

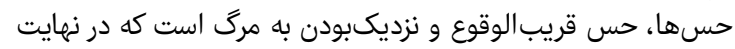

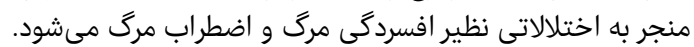

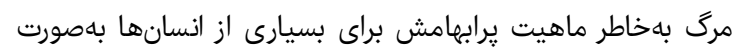

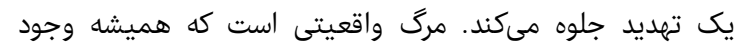

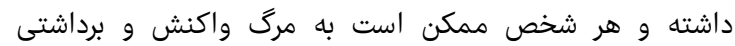

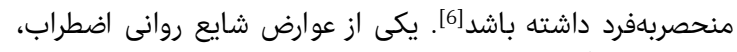

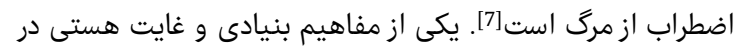

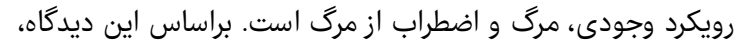

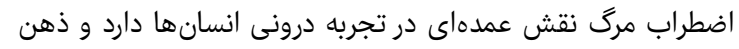

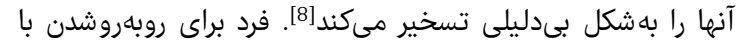

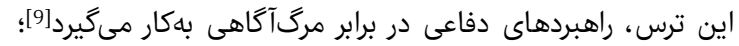

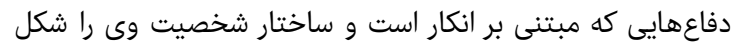

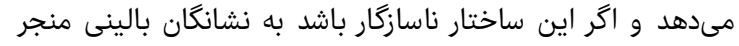

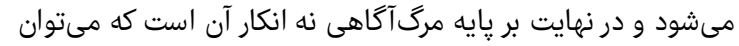

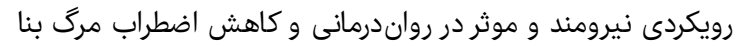

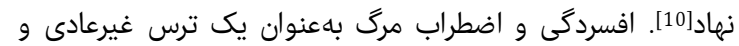

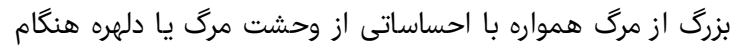

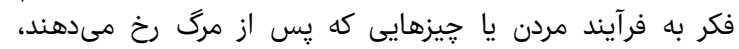

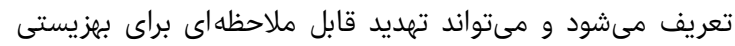
روانى بهشمار رود [11].

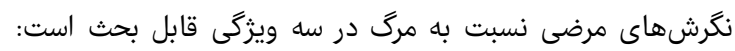

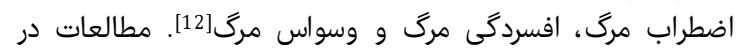

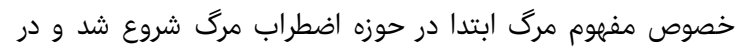

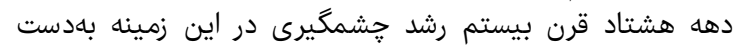

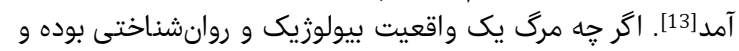

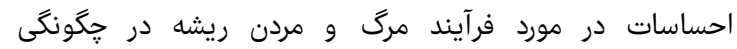

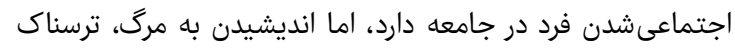

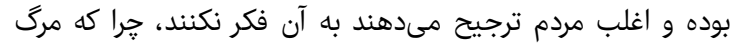

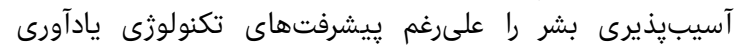

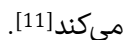
با توجه به اينكه ايران هشت سال دفاع مقدس را رُشت سر كذاشتها

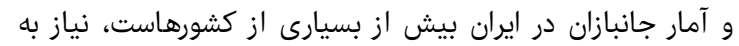

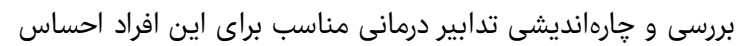

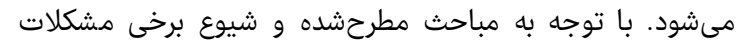

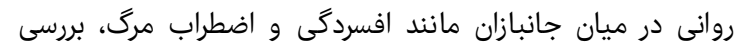

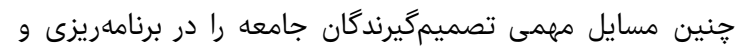

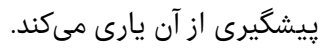
در ايران يزوهشهاى آن إرى اندكى در اين زمينه انجام شده است.

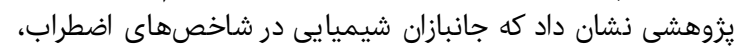

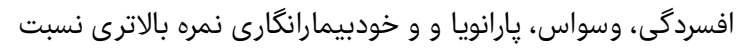

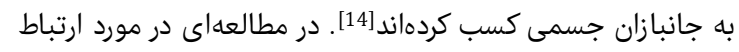

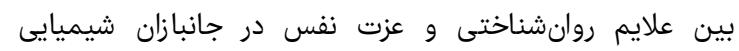

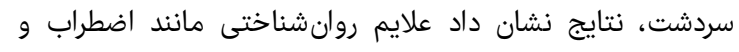

مقايسه اضطراب و افسردگى مرگ بين جانبازان

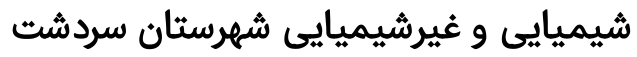

MA شهلا سليمانى كَروه روانشناسى، دانشكده علوم انسانى، واحد اروميه، دانشكاه آزاد اسلامى،

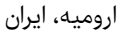

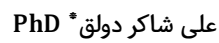

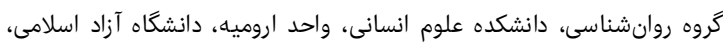

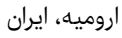

حكيده

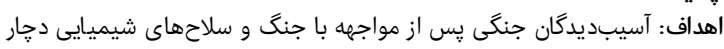

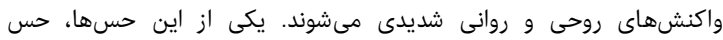

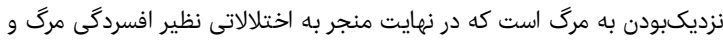

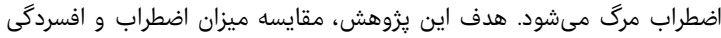

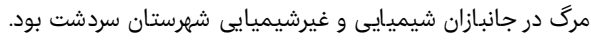

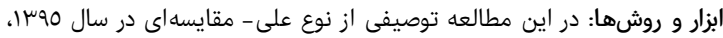

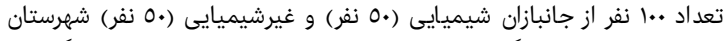

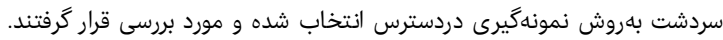

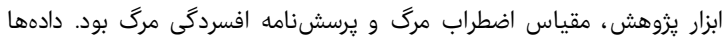

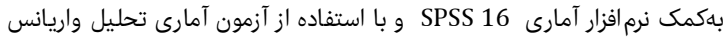

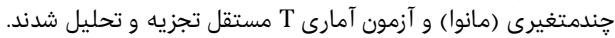

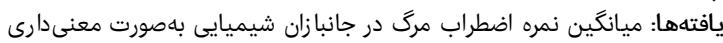

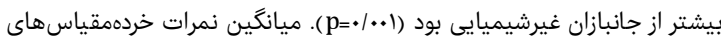

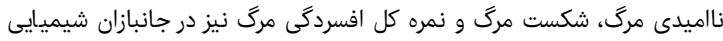

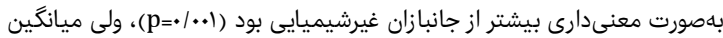

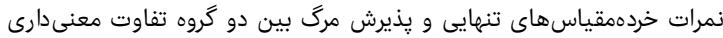

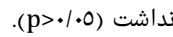

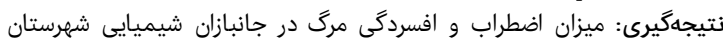
سردشت بيشتر از جانبازان غيرشيميايى إسي است.

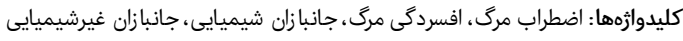

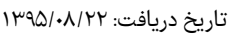

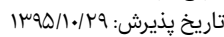
ali.shaker2000@gmail.com : نويسنده مسئول: بارئوش:

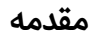

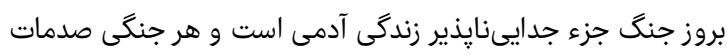

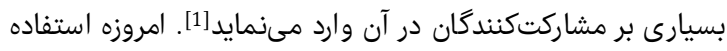

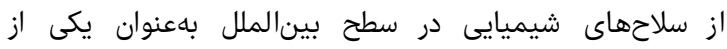

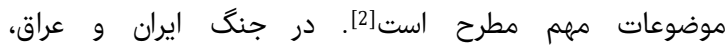

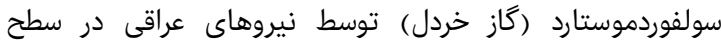

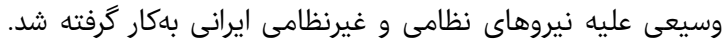

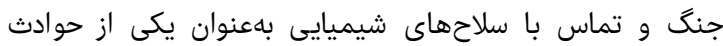

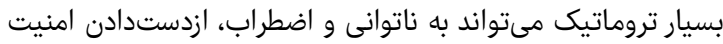

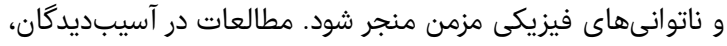

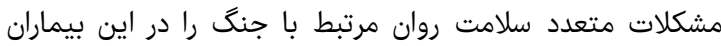

نشان مىدهند [3].

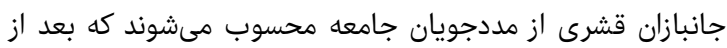

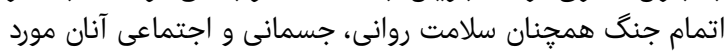

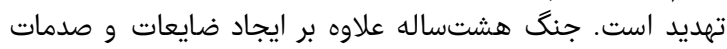

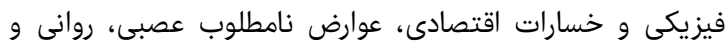

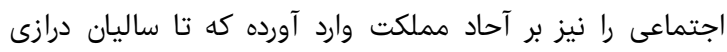

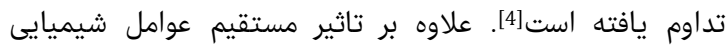

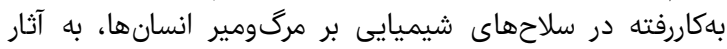

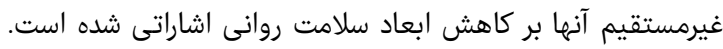

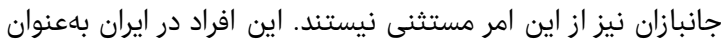

فصل نامه علمى - يخروهشى طب جانباز 


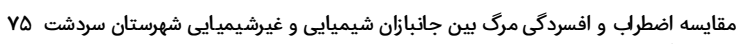

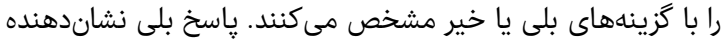

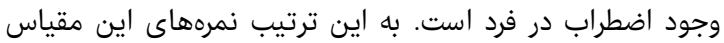

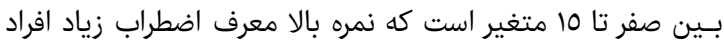

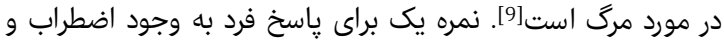

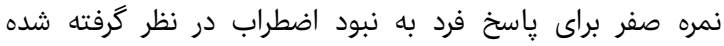

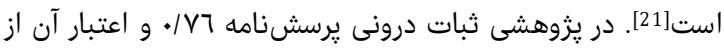

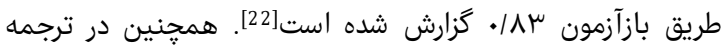

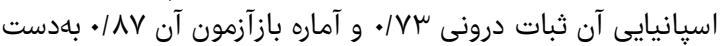

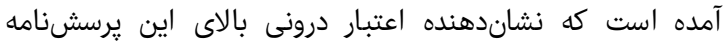

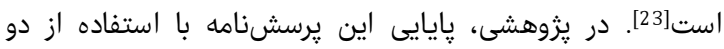

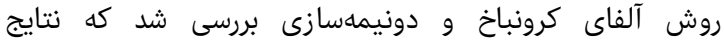

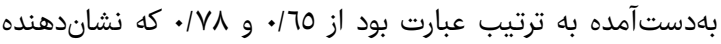

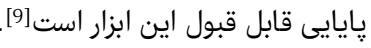

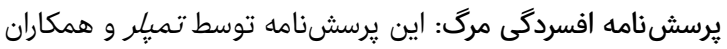

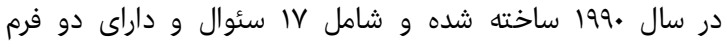

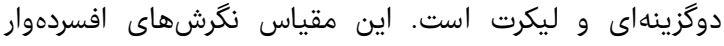

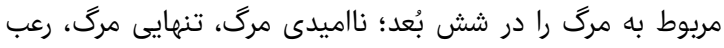

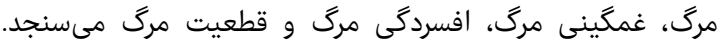

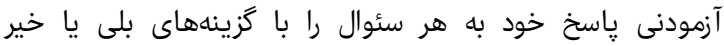

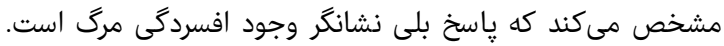

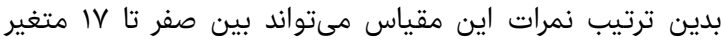

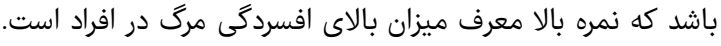

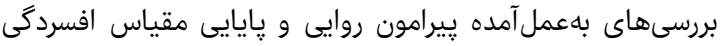

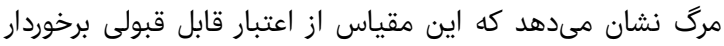

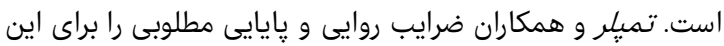
مقياس كزارش كردهاند [24].

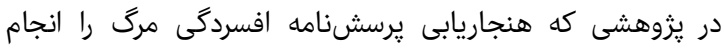

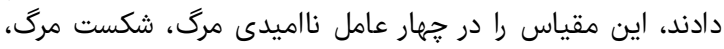

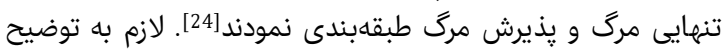

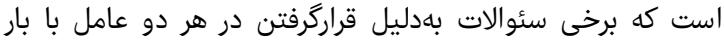

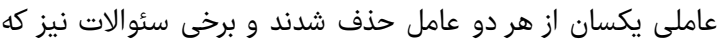

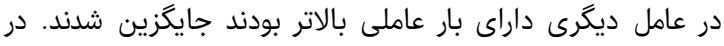

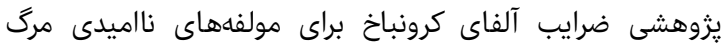

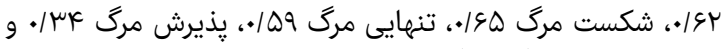

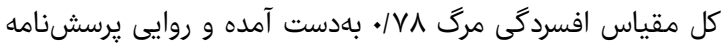

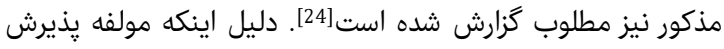

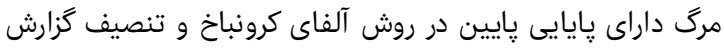

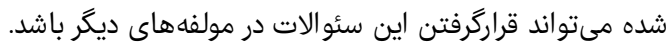

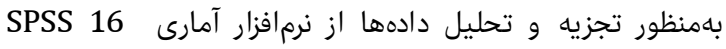

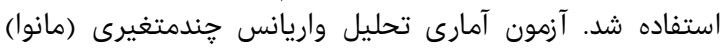

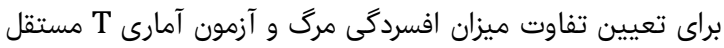

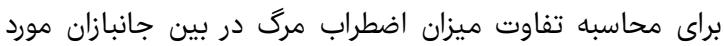

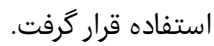

يافتهها

ميانكين نمره اضطراب مرك در جانبازان شيميايى بهصورت

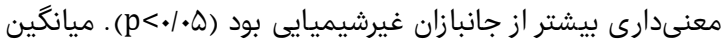

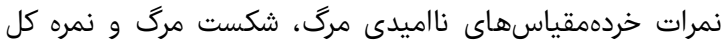

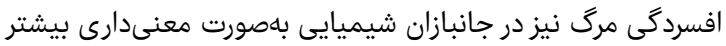

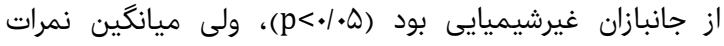

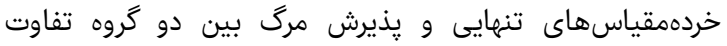

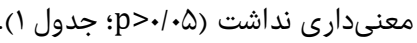

افسردگى با عزت نفس در جانبازان شيميايى رابطه معنىارى راني

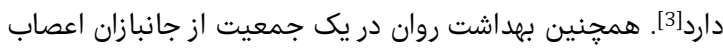

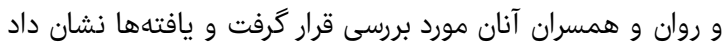

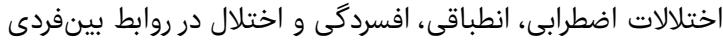

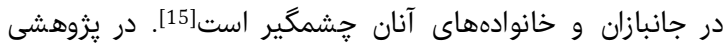

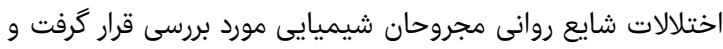

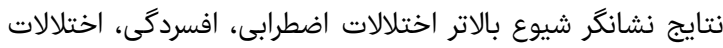

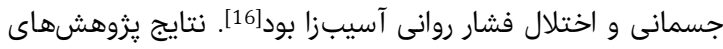

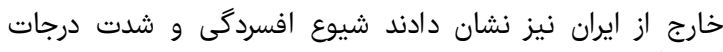

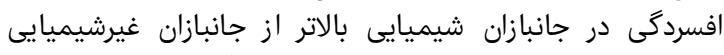

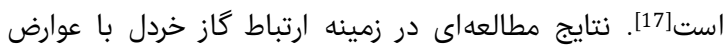

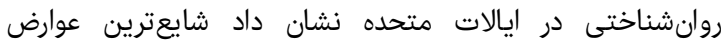

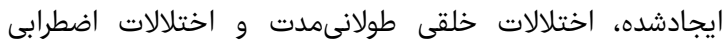

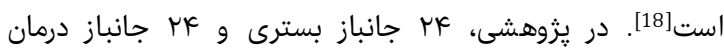

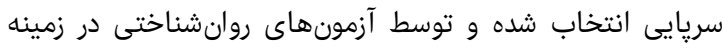

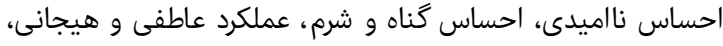

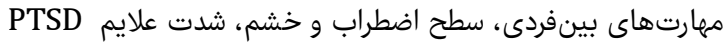

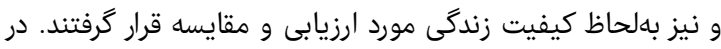

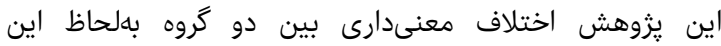

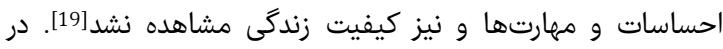

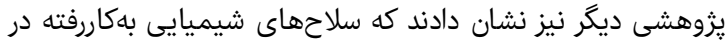

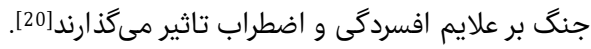

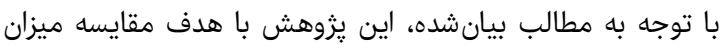

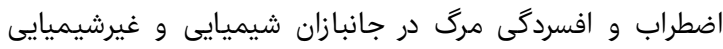

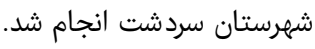

ابزار و روشها ت ابنها

اين مطالعه توصيفى از نوع على - مقايسهاى است كه در سال هوسار

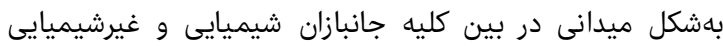

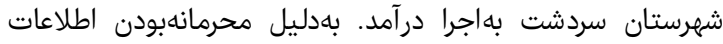

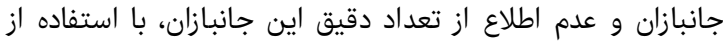

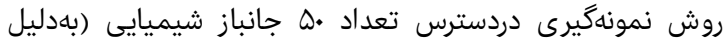

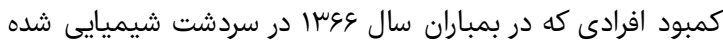

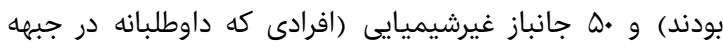

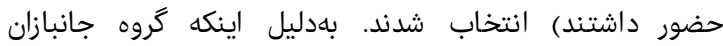

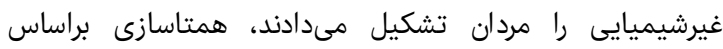

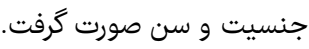

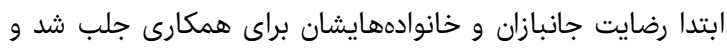

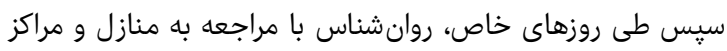

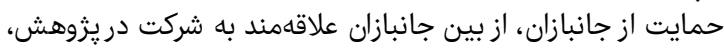

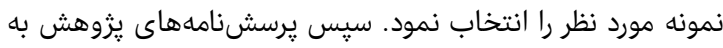

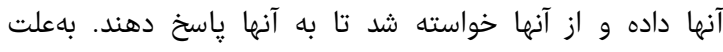

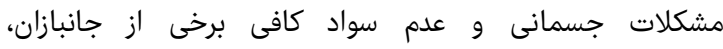

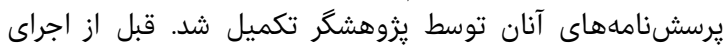

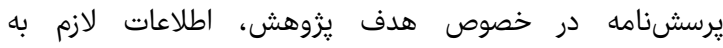
شركتكنندكان داده شد و آنها از محرمانهماندن نتايج اطمينان

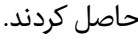
ابزار يزوهش، مقياس اضطراب مرگ و يرسشنامه افسردگى مرگ

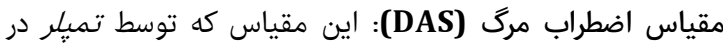

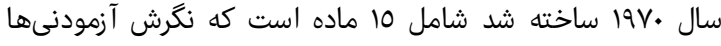

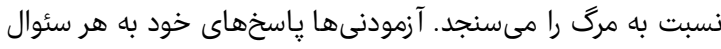


مركى موضوعى است كه هر فردى لاجرم به درجات مختلف بار با آن

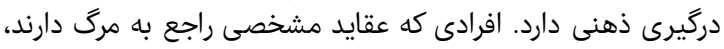

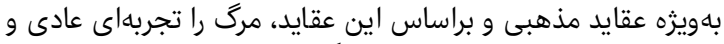

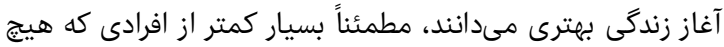

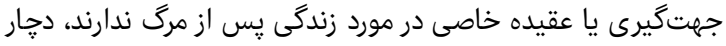

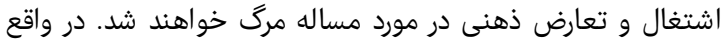

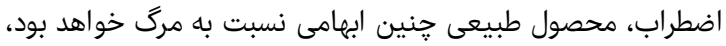

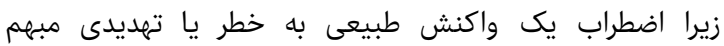

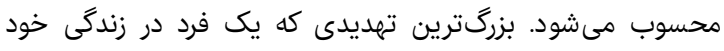

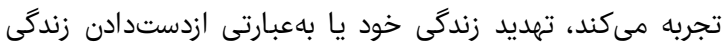

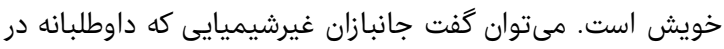

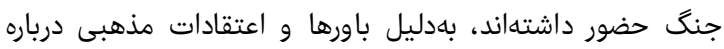

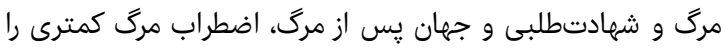

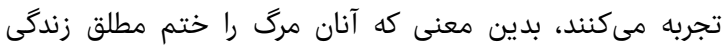
قلمداد نمىكنيد و شهيادت در راه دين دين و وطن آنان مرك را خا وظيفه شرعى و رنى

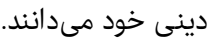
در فرضيه دوم افسردگى مركَ در جانبازان شيميايى و غيرشيميايى

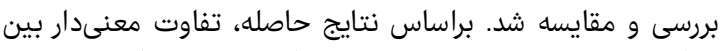

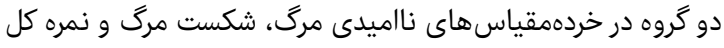

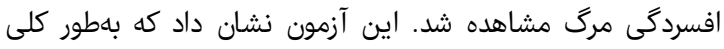

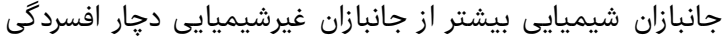

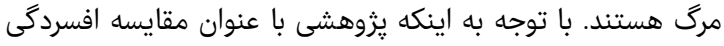

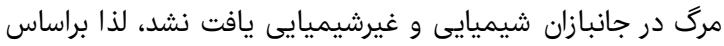

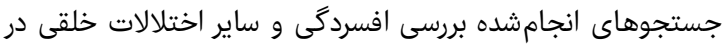

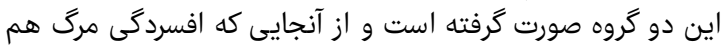

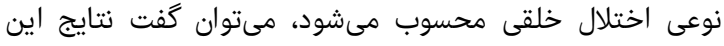

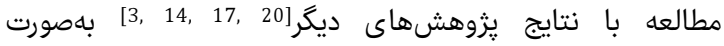

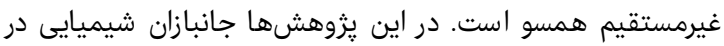

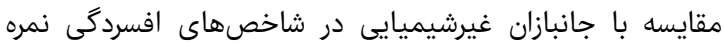

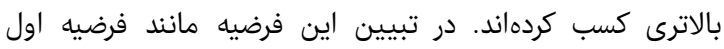

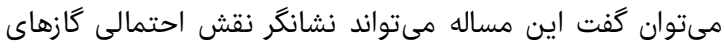

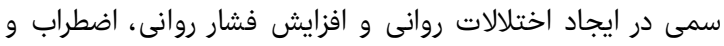

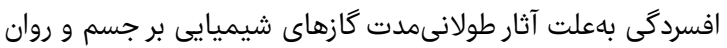

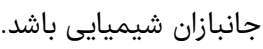
از سويى مىتوان اين تبيين بانين را اضافه نمود كه جانبازان

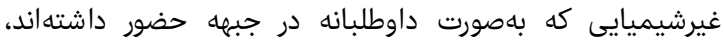

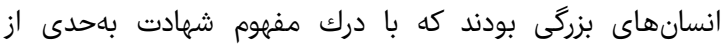

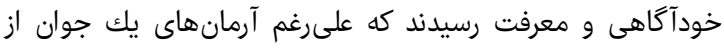

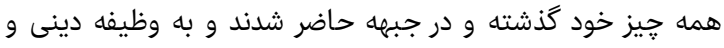

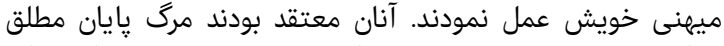

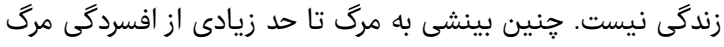

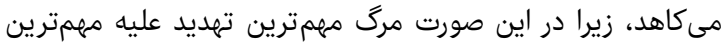

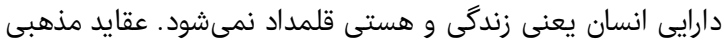

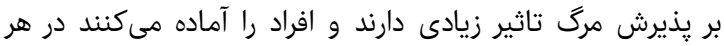
زمان آماده يذيرفتن واقعيت مرك باتير باشيند

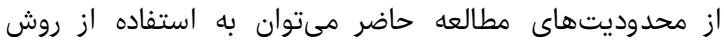

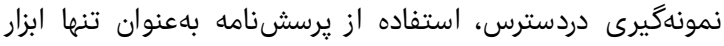

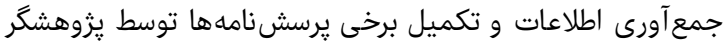

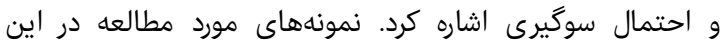

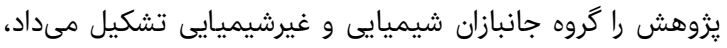

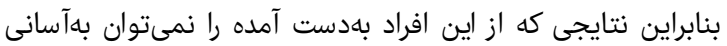

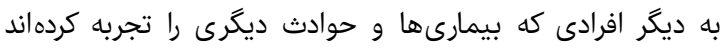

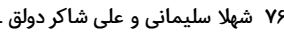

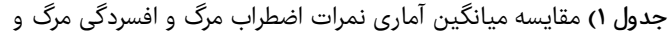

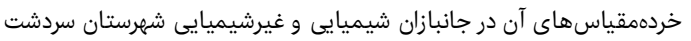

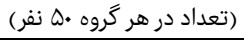

\begin{tabular}{|c|c|c|}
\hline سطح معنىداري & كروه جانبازان غيرشيميايى & كروه جانبازان شيميايى \\
\hline \multirow{3}{*}{.$/ . .1$} & & اضطراب مرگ \\
\hline & $r / V \varepsilon \pm r / r \varepsilon$ & $V / 0 \varepsilon \pm \varepsilon / .$. \\
\hline & & نااميدى مرگ \\
\hline \multirow[t]{2}{*}{.$/ . .1$} & $\cdot / 7{ }_{ \pm}^{\mu} \cdot / 7$ & 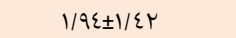 \\
\hline & & شكست مرگ \\
\hline \multirow[t]{2}{*}{.$/ . .1$} & $\cdot / V Y \pm \cdot / 7 \varepsilon$ & $r / \cdot \varepsilon \pm 1 / V r$ \\
\hline & & تنهايى مرگ \\
\hline \multirow[t]{2}{*}{.1 .71} & $r / 1 \cdot \pm 1 / \cdot \mu$ & $r / O r \pm 1 / .9$ \\
\hline & & يذيرش مرگ \\
\hline \multirow[t]{2}{*}{. 1410} & $. / 9 \Lambda \pm . / 7 \Lambda$ & $1 / . . \pm . / 79$ \\
\hline & & افسردخى مرگ \\
\hline.$/ . .1$ & $\varepsilon / r\rceil \pm r / I$ & $V / \varepsilon \wedge \pm \mu / \Lambda$. \\
\hline
\end{tabular}

\section{بحث}

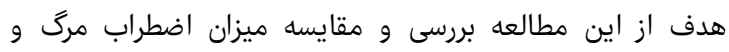

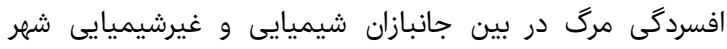

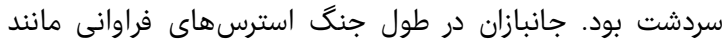

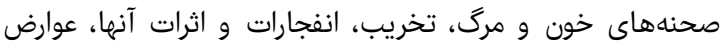

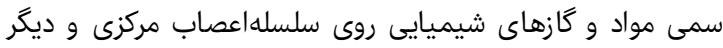

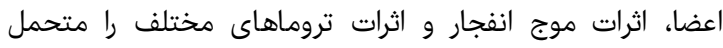

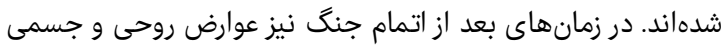

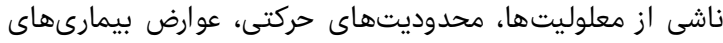

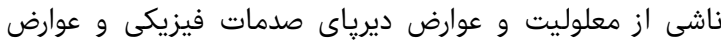

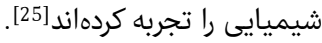

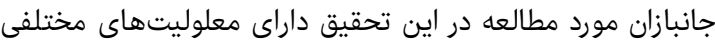

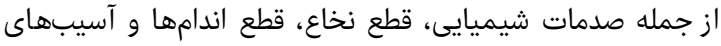

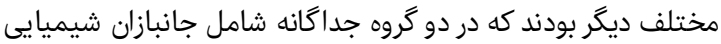

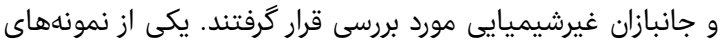

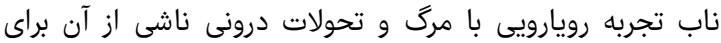

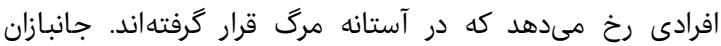

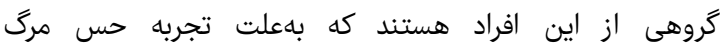

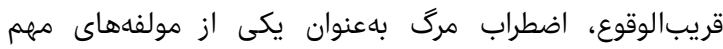
روانشناختى در آنان مطرح است.

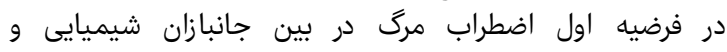

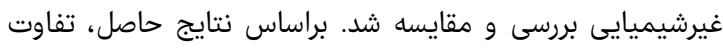

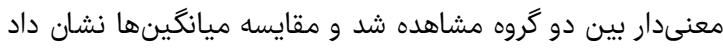

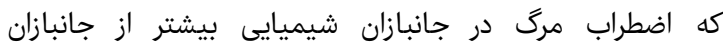

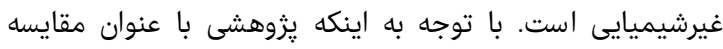

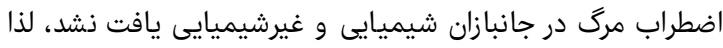

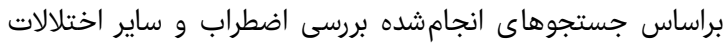

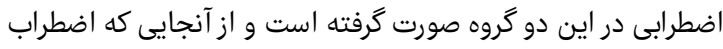

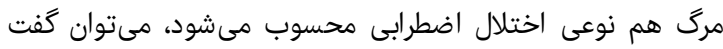

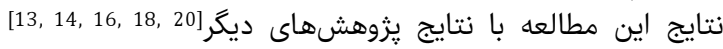

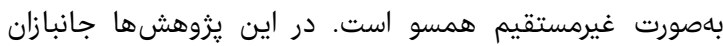

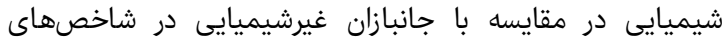

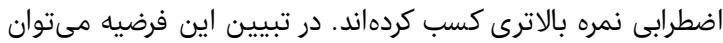

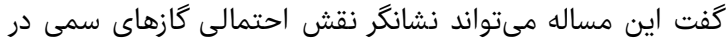

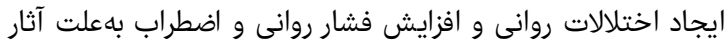

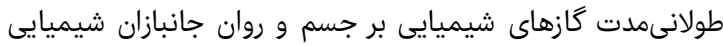


مقايسه اضطراب و افسردكى مرك يين جانبازان شيميايى و غيرشيميايى شهرستان سردشت VVIV 5- Dabbaghi P, Bolhari J. The effect of war related PTSD on wife's marital satisfaction, mental health and children's behavioral problems. Ann Mil Health Sci Res. 2009;7(1):1-7. [Persian]

6- Hoseini SM. Death. Tehran: Neyestan Book Publishing; 2004. [Persian]

7- Nyatunga B. Towards a definition of death anxiety. Int J Palliat Nurs. 2006;12(9):410-3.

8- Nourbala AA. Psychiatric Disoders in Combatants. J Mil Med. 2003;4(4):257-60. [Persian]

9- Kajbaf M, Ghasemiannezhad Jahromi AN, Ahmadi Foroshani SH. Spiritual effect of group therapy on depression, anxiety, death and belief in life after death continue to rely on the reports of the death of the student experience. Stud Appl Psychol. 2015;16(4):4-13. [Persian]

10- Yalom A. Existential psychotherapy. Habib S, translator. Tehran: Ney Publishing; 2011. [Persian]

11- Ghorbani E, Saadatmand S, Sepehrian Azar F, Asadnia S, Feyzipour H. Surveying the relationship between hope, death, anxiety with mental health on students of urmia university. Urmia Med J. 2013;28(4):607-16. [Persian]

12- Buzzanga V, Miller H, Perne S. The relation between death anxiety and levelof self-esteem: A reassessment. Bull Psychon Soc. 1989;27(6):570-2.

13- Mohammadzade A, Najafi M. he Prediction of Death Obsession According to Religiosity Orientations: Role of sex. Iran J Clin Psychol. 2010;2(3):65-72. [Persion]

14- Behdani F, Fayyazi Bordbar MR, Hebrani P, Talebi M, Kohestani L. Psychiatric symptoms in chemical and somatic combat veterans. J Mil Med. 2009;11(1):31-5. [Persion]

15- Bahreinian SA, Borhani H. Mental health in group of war veterans and their spouses in Qom. Res Med. 2003;27(4):305-12. [Persian]

16- Tavallaie SA, Assari SH, Najafi M, Habibi M, Ghanei M. Study of sleep quality in chemical-warfare-agents exposed veterans. J Mil Med. 2005;6(4):241-8. [Persian]

17- Vafaei B, Seydi A. Study of the prevalence and intensity of depression in 100 devotees with chemical and non-chemical war injuies (30-70\%) of Imposed war in Tabriz. J Mil Med. 2003;5(2):105-10. [Persian

18- Pechura CM, Rall DP. Relationship of mustard agent and lewisite exposure to psychological dysfunction. In: Veterans at risk: The health effects of mustard gas and lewisite. Pechura CM, Rall DP, editors. Washington DC: National Academies Press; 1993. pp. 199-213.

19- Ragsdale KG, Cox RD, Finn P, Eisler RM. Effectiveness of short-term specialize inpatienttreatment for warrelated posttraumatic stress disorder: A role for advanture-based counseling and psychodrama. J Trauma Stress. 1996; 9(2):269-83.

20- Hashemian F, Khoshnood K, Desai M, Falahati F, Kasl S, Southwick S. Anxiety, depression, and posttraumatic stress in iranian survivors of chemical warfare. JAMA. 2006;296(5):560-6.

21- Masoudzade A, Setare J, Mohammadpour Tahamtan RA, Modanlou Kordi M. A survey of death anxiety among personnel of a hospital in Sari. J Mazandaran Univ Med Sci. 2006;18(67):84-90. [Persian]

22- Brewer G. Differing death scenarios: self- esteem and Death anxiety [Dissertation]. Johnson City, Tennessee: East Tennessee State University; 2002.

23- Tomás-Sábado J, Gómez-Benito J. Psychometric properties of the Spanish form of Templer's Death Anxiety scale. Psychol Rep. 2002;91(3 Pt 2):1116-20.
تعميم داد. از محدوديتهاى ديگر اين مطالعه مىتوان به عدم إندائ

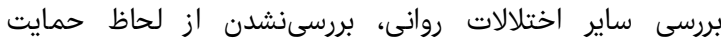

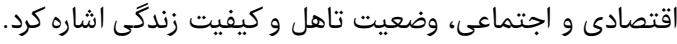

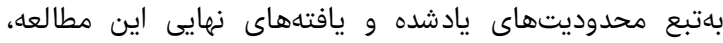

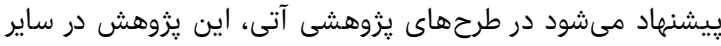

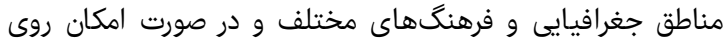

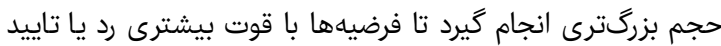

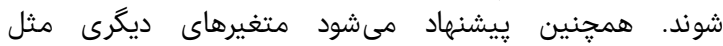

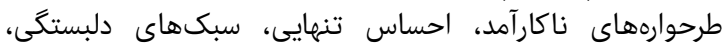

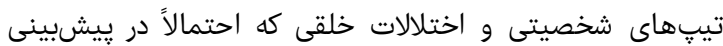

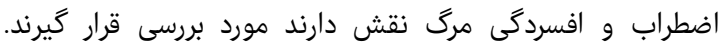

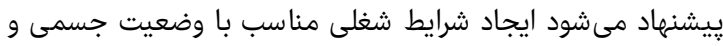

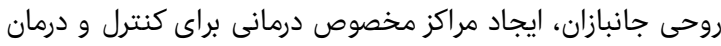

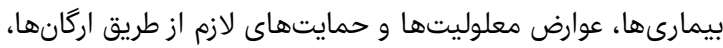

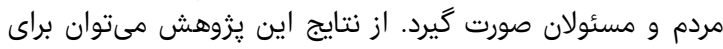

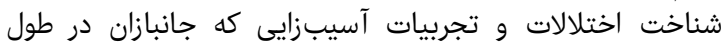

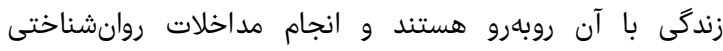
بهمنظور بهبود سلامت روان اين افراد استفاده نمود.

$$
\begin{aligned}
& \text { نتيجهگيرى } \\
& \text { ميزان اضطراب و افسردگى مرگ در جانيرى جانبازان شيميايى شهرستان }
\end{aligned}
$$

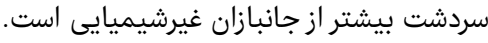

تشكر و قدردانى: بدون همكارى جانبازان محترم و خانوادههاى آنها

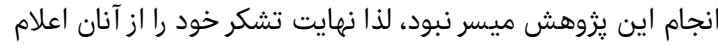

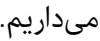

تاييديه اخلاقى: در ابتدا رضايت جانبازان و خانوادههايشان براى

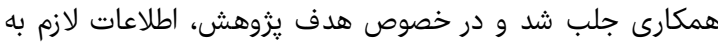

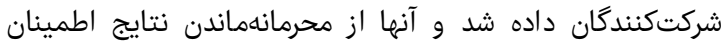

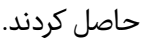
تعارض منافع: موردى از سوى نويسندگان بيان نشده است.

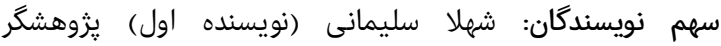

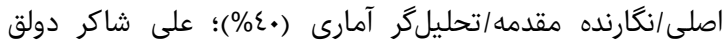

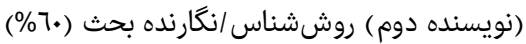
منابع مالى: موردى از سوى نويسنداس اندارنان بيان نشده است.

\section{منابع}

1- Mehrabi H, Norouzi S, Mirzaei Gh, Kazemi H. An investigation the relationship between post traumatic growth and attachment styles, stress coping styles \& quality of life in veterans with post-traumatic stress disorder. J Nurs Physicians Combat. 2015;2(4):153-61. [Persian]

2- Mohaghegh Motlagh SJ, Momtazi S, Mousavi Nasab SN, Arab A, Sabouri E, Sabouri A. Post-traumatic stress disorder in male chemical injured war veterans compared to non-chemical war veterans. Med J Mashhad Univ Med Sci. 2014;56(6):361-8. [Persian]

3- Fathi Ashtiyani A, Toulayi SA, Azizabadi Farahani M, Moghani Lankarani, A. Association of psychological symptoms and self esteem in chemical warfare agent exposed veterans. J Mil Med. 2008;9(4):273-82. [Persian] 4- Karami GR, Amiri M, Ameli J, Kachooei H, Ghodoosi K, Saadat AR, et al. Psychological health status of mustard gas exposed veterans. J Mil Med. 2006;8(1):1-7. [Persian] 
شهيلا سليمانى و على شاكر دولق VA VA

treatment plan wives of veterans with PTSD impact on reducing symptoms of this disorder in veterans with Adler's approach and improve marital satisfaction. Psychol Stud. 2008;4(3):93-114. [Persian]
24- Mohammadzade A, Rezaie A, Aghazade SE. Death Depression scale validation in students. J Ilam Univ Med Sci. 2016;1(24):89-97. [Persian]

25- Alizade $H$, Hashemi M. The effectiveness of the 